\title{
Blinded 12-week comparison of once-daily indacaterol and tiotropium in COPD
}

\author{
R. Buhl*, L.J. Dunn" ${ }^{\#}$ C. Disdier ${ }^{\oplus}$, C. Lassen ${ }^{+}$, C. Amos ${ }^{+}$, M. Henley ${ }^{+}$and \\ B. Kramer ${ }^{\S}$, on behalf of the INTENSITY study investigators
}

ABSTRACT: Two, once daily (q.d.) inhaled bronchodilators are available for the treatment of chronic obstructive pulmonary disease (COPD): the $\beta_{2}$-agonist indacaterol and the anticholinergic tiotropium. This blinded study compared the efficacy of these two agents and assessed their safety and tolerability.

Patients with moderate-to-severe COPD were randomised to treatment with indacaterol $150 \mu \mathrm{g}$ q.d. ( $n=797)$ or tiotropium $18 \mu \mathrm{g}$ q.d. $(\mathrm{n}=801)$ for 12 weeks.

After 12 weeks, the two treatments had similar overall effects on "trough" (24 h post-dose) forced expiratory volume in $1 \mathrm{~s}$. Indacaterol-treated patients had greater improvements in transition dyspnoea index (TDI) total score (least squares means 2.01 versus $1.43 ; p<0.001$ ) and St George's Respiratory Questionnaire (SGRQ) total score (least squares means 37.1 versus 39.2; $\mathrm{p}<0.001$; raw mean change from baseline $\mathbf{- 5 . 1}$ versus $\mathbf{- 3 . 0}$ ), and were significantly more likely to achieve clinically relevant improvements in these end-points (indacaterol versus tiotropium odds ratios of 1.49 for TDI and 1.43 for SGRQ, both $p<\mathbf{0 . 0 0 1 )}$. Adverse events were recorded for $39.7 \%$ and $37.2 \%$ of patients in the indacaterol and tiotropium treatment groups, respectively. The most frequent adverse events were COPD worsening, cough and nasopharyngitis.

Both bronchodilators demonstrated spirometric efficacy. The two treatments were well tolerated with similar adverse event profiles. Compared with tiotropium, indacaterol provided significantly greater improvements in clinical outcomes.

KEYWORDS: Chronic obstructive pulmonary disease, indacaterol, tiotropium

D) ronchodilators are central to the symptomatic management of patients with chronic obstructive pulmonary disease (COPD) [1]. While short-acting bronchodilators are useful for acute symptom relief as needed, inhaled long-acting bronchodilators are recommended as first-line maintenance treatment for patients with moderate or more severe COPD on the basis of long-term improvements that can be achieved in clinical outcomes such as dyspnoea, health status and exacerbations [1]. Two, once daily (q.d.) inhaled bronchodilators are now available: the anticholinergic tiotropium and, more recently, indacaterol, a long-acting (24 h) $\beta_{2}$-agonist. Indacaterol is approved for use in many countries, including the European Union, at doses of 150 and $300 \mu \mathrm{g}$ q.d.

Two clinical studies comparing indacaterol with tiotropium have been reported to date. In a 26week study, DONOHUE et al. [2] demonstrated that indacaterol was at least as effective a bronchodilator as tiotropium and was similarly or more effective for a number of symptomatic and health status end-points. Tiotropium treatment was not blinded in that study [2]. A short-term blinded comparison of indacaterol with tiotropium supported the findings of DONOHUE et al. [2] of similar bronchodilator efficacy [3]. Both studies were placebo controlled. Our study is the first blinded study designed with the primary endpoint of comparing indacaterol with tiotropium; the aim was to demonstrate that indacaterol had a similar, and potentially superior, efficacy profile compared with tiotropium over 12 weeks of treatment. Safety and tolerability were also assessed.

\section{METHODS}

\section{Patients}

The study enrolled male and female adults $\geqslant 40$ yrs of age, with a diagnosis of moderate-tosevere COPD (post-bronchodilator (salbutamol $400 \mu \mathrm{g}$ ) forced expiratory volume in $1 \mathrm{~s}$ (FEV1) $<80 \%$ and $\geqslant 30 \%$ predicted, FEV1/forced vital capacity (FVC) $<70 \%$ ) [4] and a smoking history of $\geqslant 10$ pack-yrs. Patients with a history of asthma, or a recent COPD exacerbation or respiratory infection, were not included. The patients gave
AFFILIATIONS

*Pulmonary Dept, Mainz University Hospital, Mainz, Germany.

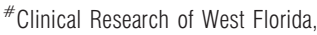
Clearwater, FL,

${ }^{\S}$ Respiratory Development, Novartis Pharmaceuticals Corporation, East Hanover, NJ, USA.

- San Pedro De Alcantara Hospital, Caceres, Spain.

${ }^{+}$Novartis Horsham Research Centre, Horsham, UK.

CORRESPONDENCE

R. Buhl

Pulmonary Dept

Mainz University Hospital

Langenbeckstrasse 1

Mainz

D-55131

Germany

E-mail: Roland.Buhl@unimedizin-

mainz.de

Received:

Dec 132010

Accepted after revision:

April 242011

First published online:

May 262011 
their written informed consent before any study procedure was performed.

\section{Study design}

This was a 12-week, multicentre, randomised, parallel-group, blinded, double-dummy study. The protocol was approved by the institutional review boards or ethics committees at each of the participating centres (respiratory out-patient clinics, physician's offices and clinical research centres).

\section{Study medications}

Following a 2-week run-in, patients were randomised to treatment with indacaterol $150 \mu \mathrm{g}$ q.d. via single-dose dry powder inhaler (Onbrez $₫$ Breezhaler ${ }$; Novartis, Basel, Switzerland) or tiotropium $18 \mu \mathrm{g}$ q.d. via its proprietary single-dose dry powder inhaler (Spiriva ${ }^{\circledR}$ HandiHaler $®$; Boehringer Ingelheim, Ingelheim, Germany). Patients receiving indacaterol also took a placebo via the inhaler used for tiotropium, and patients receiving tiotropium took a placebo via the inhaler used for indacaterol. Blinding was achieved by specifying that study medications were dispensed by a third party not involved in other aspects of the study.

Patients were also given a short-acting $\beta_{2}$-agonist (salbutamol) to use as required to relieve symptoms. Apart from study treatments, no other bronchodilator use was permitted. Longacting bronchodilators were discontinued prior to the run-in with an appropriate washout: 7 days for tiotropium or theophylline and 2 days for salmeterol or formoterol. Patients previously receiving inhaled corticosteroid (ICS) monotherapy continued this treatment unchanged; any patients on a fixeddose ICS and $\beta_{2}$-agonist combination were switched to the ICS component at an equivalent dose and regimen.

\section{Objectives, assessments and outcome measures}

The primary objective was to demonstrate non-inferiority of indacaterol to tiotropium in their effect on "trough" FEV1 (mean of $23 \mathrm{~h} 10 \mathrm{~min}$ and $23 \mathrm{~h} 45 \mathrm{~min}$ post-dose measurements) after 12 weeks of treatment. For each assessment, three acceptable manoeuvres were performed and the highest values of FEV1 and FVC were recorded. Spirometry was performed according to recognised standards [5], with the same personnel and equipment for each patient during the study as far as possible. All sites were supplied with the same make and model of spirometer (Vitalograph 6800; Vitalograph Maids Moreton, UK), and all persons performing the spirometry testing were certified in the use of the equipment before use. In addition, all spirometry assessments were reviewed centrally to ensure the manoeuvres met the standards for acceptability and repeatability.

If non-inferiority for trough FEV1 was shown, the comparison was also to be tested for superiority of indacaterol to tiotropium. Secondary end-points were as follows. FEV1 and FVC were measured at other time points. Dyspnoea was assessed by the transition dyspnoea index (TDI) total score at week 12 ( $\geqslant 1$ point is a clinically relevant change from baseline) $[6,7]$. Health status was assessed by St George's Respiratory Questionnaire (SGRQ) score after 12 weeks ( $\geqslant 4$ units is a clinically relevant change from baseline) [8-10]. The use of as-needed ("rescue") salbutamol over 12 weeks was recorded daily by patients in an electronic diary. The diary was also used to record the percentages of days with no COPD symptoms, nights with no awakenings and days of usual activities.
Safety and tolerability were assessed by adverse events and the incidence of notable values for vital signs, Fridericia's correction of QT interval (QTc interval) measured from ECGs, reduced levels of serum potassium $\left(<3.0 \mathrm{mmol} \cdot \mathrm{L}^{-1}\right)$ and elevated blood glucose $\left(>9.99 \mathrm{mmol} \cdot \mathrm{L}^{-1}\right)$ measured at any time post-baseline (blood samples were taken pre-dose and ECG and vital signs measured $30 \mathrm{~min}$ post-dose after 4 and 12 weeks).

\section{Randomisation and blinding}

Patients were randomised in a 1:1 ratio, and stratified by smoking status (current/ex-smoker). The order of use of the inhalers was randomly assigned. The assigned study treatment was dispensed to patients by a third party who was not otherwise involved in the study. Patients, who were blinded to treatment assignment, self-administered their treatment at each visit. Investigators, study staff performing the assessments and data analysts were blinded and did not observe the actual treatment patients took at clinic visits.

\section{Statistical methods}

Three patient populations were defined for analysis. 1) The full analysis (intention-to-treat) population comprised randomised patients who received at least one dose of study drug and who were analysed according to the allocated treatment group. 2) The per-protocol population included patients in the full analysis population who did not have major protocol deviations and who were analysed according to treatment received. 3) The safety population comprised patients who received at least one dose of study drug and who were analysed according to treatment received.

The primary variable was analysed using a mixed-model ANCOVA with treatment, smoking status and country as fixed effects, centre nested within country as a random effect, and baseline FEV1, FEV1 reversibility components (assessed at screening) and ICS use as covariates. Missing values were imputed by carrying forward the last observation. Data are presented as least squares mean (LSM) with standard errors for group mean values and 95\% confidence intervals (CI) for the difference between treatments. Non-inferiority of indacaterol to tiotropium was demonstrated if the $95 \% \mathrm{CI}$ for the mean FEV1 difference of indacaterol minus tiotropium was entirely to the right of (higher than) $-55 \mathrm{~mL}$ in the per-protocol population. If non-inferiority was determined, superiority could be demonstrated in the full analysis population if (in the full analysis population) the p-value was $<0.05$ (two-sided) and the $95 \%$ CI was entirely to the right of $0 \mathrm{~mL}$. In a preplanned analysis, the primary variable was also compared in subgroups of patients categorised according to their baseline age, sex, smoking history, COPD severity, ICS use and salbutamol FEV1 reversibility.

The secondary variables were analysed using a mixed model similar to that used for the primary end-point, although for the full analysis set, with appropriate baseline measurements as covariates and without adjustment for multiplicity. Values for missing TDI and SGRQ total scores were imputed by carrying forward the last observation. The proportions of patients achieving a clinically important improvement in TDI total scores and SGRQ total scores were analysed using a logistic regression model with terms for treatment, smoking status and country as fixed effects, centre nested within country as a 
random effect, and with FEV1 reversibility, ICS use and baseline dyspnoea index or SGRQ scores as covariates. Results are given as estimated adjusted odds ratios with $95 \%$ CIs and two-sided p-values. Results were also summarised as unadjusted (raw) mean changes from baseline. Data for the exploratory variables of diary records (percentages of days with no COPD symptoms, nights with no awakenings and days of usual activities) were summarised and not analysed statistically.

Previous study results provided an assumed treatment difference (indacaterol-tiotropium) of $40 \mathrm{~mL}$ for trough FEV1 at week 12 [2], with a standard deviation of $225 \mathrm{~mL}[2,11,12]$. A sample size of 666 evaluable patients per treatment group was needed to detect this difference as statistically significant at the $5 \%$ level (two-sided) with $90 \%$ power. This sample size provided $>99 \%$ power for detecting non-inferiority at the $2.5 \%$ significance level (one-sided), assuming a non-inferiority margin of $55 \mathrm{~mL}$ (this being half the CI associated with the published treatment difference between tiotropium and placebo) [13]. An assumed drop-out rate of $15 \%$ gave a minimum sample size of 1,568 patients.

\section{RESULTS}

The disposition of patients is shown in table 1, with baseline demographics and other characteristics presented in table 2. Most patients (95\%) were Caucasian. Most patients had moderate $(62 \%)$ or severe COPD $(37 \%)$. All calculations to determine the severity of COPD in patients at screening were performed at study centres. After database lock, COPD severity was derived using a standardised statistical calculation. This resulted in one patient (in the tiotropium group) being re-classified as having mild COPD, 13 as having very severe COPD (five in the indacaterol group and eight in the

\section{TABLE 1 Disposition of patients}

\begin{tabular}{lccc} 
& Indacaterol & Tiotropium & Total \\
\hline Screened $\mathbf{n}$ & & & \\
Randomised & $797(100.0)$ & $801(100.0)$ & $1598(100.0)$ \\
Exposed & $794(99.6)$ & $799(99.8)$ & $1593(99.7)$ \\
Completed & $737(92.5)$ & $740(92.4)$ & $1477(92.4)$ \\
Discontinued & $60(7.5)$ & $61(7.6)$ & $121(7.6)$ \\
$\quad$ Adverse event(s) & $31(3.9)$ & $27(3.4)$ & $58(3.6)$ \\
$\quad$ Subject withdrew consent & $8(1.0)$ & $7(0.9)$ & $15(0.9)$ \\
$\quad$ Protocol deviation & $8(1.0)$ & $11(1.4)$ & $19(1.2)$ \\
$\quad$ Administrative problems & $5(0.6)$ & $4(0.5)$ & $9(0.6)$ \\
$\quad$ Abnormal test procedure result(s) & $3(0.4)$ & $0(0.0)$ & $3(0.2)$ \\
$\quad$ Abnormal laboratory value(s) & $2(0.3)$ & $4(0.5)$ & $6(0.4)$ \\
$\quad$ Lost to follow-up & $2(0.3)$ & $3(0.4)$ & $5(0.3)$ \\
$\quad$ Unsatisfactory therapeutic effect & $1(0.1)$ & $3(0.4)$ & $4(0.3)$ \\
$\quad$ Death & $0(0.0)$ & $2(0.2)$ & $2(0.1)$ \\
Analysed for safety & $794(99.6)$ & $799(99.8)$ & $1593(99.7)$ \\
Analysed for efficacy, & $794(99.6)$ & $799(99.8)$ & $1593(99.7)$ \\
$\quad$ full analysis set & & & \\
Analysed for efficacy, & & & \\
$\quad$ per-protocol set & $599(75.2)$ & $624(77.9)$ & $1223(76.5)$ \\
\hline & & & \\
\hline
\end{tabular}

Data are presented as $n(\%)$, unless otherwise stated tiotropium group), and two as having a post-bronchodilator FEV1/FVC ratio $\geqslant 70 \%$ (both in the indacaterol group).

\section{Spirometry}

Trough FEV1 at week 12 was $1.44 \mathrm{~L}$ and $1.43 \mathrm{~L}$ with indacaterol and tiotropium, respectively. The CIs for the rounded treatment difference $(0 \mathrm{~mL} ; 95 \% \mathrm{CI}-20-20 \mathrm{~mL})$ in the per-protocol population met the pre-defined criteria for non-inferiority $(p<0.001)$. Subsequent criteria for superiority in terms of trough FEV1 at week 12 were not met. The corresponding raw mean changes from baseline in trough FEV1 were $130 \mathrm{~mL}(11.1 \%)$ and $120 \mathrm{~mL}$ (10.6\%) with indacaterol and tiotropium, respectively.

Trough FEV1 at week 12 in patient subgroups of the intent-totreat population, analysed according to baseline age, sex, smoking history, COPD severity, ICS use and salbutamol reversibility, is shown in table 3 .

A formal calculation of trough FVC was not made. However, for FVC values at $23 \mathrm{~h} 10 \mathrm{~min}$ and $23 \mathrm{~h} 45 \mathrm{~min}$ post-dose at week 12 (the two time-points used to determine trough FEV1), there was no difference between treatments, with values of 2.83 and $2.84 \mathrm{~L}$ at $23 \mathrm{~h} 10 \mathrm{~min}$ for indacaterol and tiotropium, respectively, and 2.89 and $2.90 \mathrm{~L}$ at $23 \mathrm{~h} 45 \mathrm{~min}$ post-dose, respectively.

At 5 min after the first dose on day 1, FEV1 was LSM $70 \mathrm{~mL}$ (95\% CI 60-80) higher with indacaterol than with tiotropium $(p<0.001)$. The treatment difference remained statistically significant at $30 \mathrm{~min}$ (LSM $30 \mathrm{~mL}, 95 \%$ CI 20-40, p<0.001) and $1 \mathrm{~h}$ (LSM $20 \mathrm{~mL}, 95 \%$ CI 0-30, p<0.01) post-dose. FVC followed a similar pattern and was significantly higher with indacaterol than with tiotropium at post-dose time-points of $5 \mathrm{~min}$ (LSM $120 \mathrm{~mL}, 95 \%$ CI 100-140, p<0.001), 30 min (LSM $40 \mathrm{~mL}, 20-70, \mathrm{p}<0.001$ ), and $1 \mathrm{~h}$ and $2 \mathrm{~h}$ (both LSM $30 \mathrm{~mL}, 10$ $60, \mathrm{p}<0.05)$. The adjusted between-treatment differences at

\section{TABLE 2 Demographics and baseline characteristics}

\begin{tabular}{lcc} 
& Indacaterol & Tiotropium \\
\hline Subjects n & 794 & 799 \\
Age yrs & $63.6 \pm 8.60$ & $63.4 \pm 8.29$ \\
Male/female & $70 / 30$ & $67 / 33$ \\
Duration of COPD yrs & $7.0 \pm 6.01$ & $7.0 \pm 6.32$ \\
ICS use & 54 & 56 \\
Ex-smoker/smoker & $55 / 45$ & $56 / 44$ \\
Smoking history pack-yrs & $43.2 \pm 20.87$ & $41.8 \pm 19.81$ \\
FEV1 post-bronchodilator L & $1.53 \pm 0.459$ & $1.52 \pm 0.447$ \\
FEV reversibility & $14.1 \pm 12.63$ & $13.7 \pm 13.44$ \\
FEV 1 \% pred post-bronchodilator & $54.6 \pm 12.80$ & $54.3 \pm 12.81$ \\
FEV /FVC post-bronchodilator & $51.0 \pm 9.38$ & $51.2 \pm 9.42$ \\
Use of as-needed salbutamol puffs $\cdot$ day $^{-1}$ & $3.8 \pm 3.74$ & $3.6 \pm 3.51$ \\
BDI score & $6.8 \pm 2.2$ & $6.8 \pm 2.23$ \\
SGRQ score & $42.3 \pm 17.60$ & $42.7 \pm 18.04$ \\
\hline
\end{tabular}

Data are presented as $\%$ or mean \pm SD, unless otherwise stated. COPD: chronic obstructive pulmonary disease; ICS: inhaled corticosteroid; FEV1: forced expiratory volume in $1 \mathrm{~s}$; \% pred: \% predicted; FVC: forced vital capacity; BDI: baseline dyspnoea index; SGRQ: St George's Respiratory Questionnaire. 


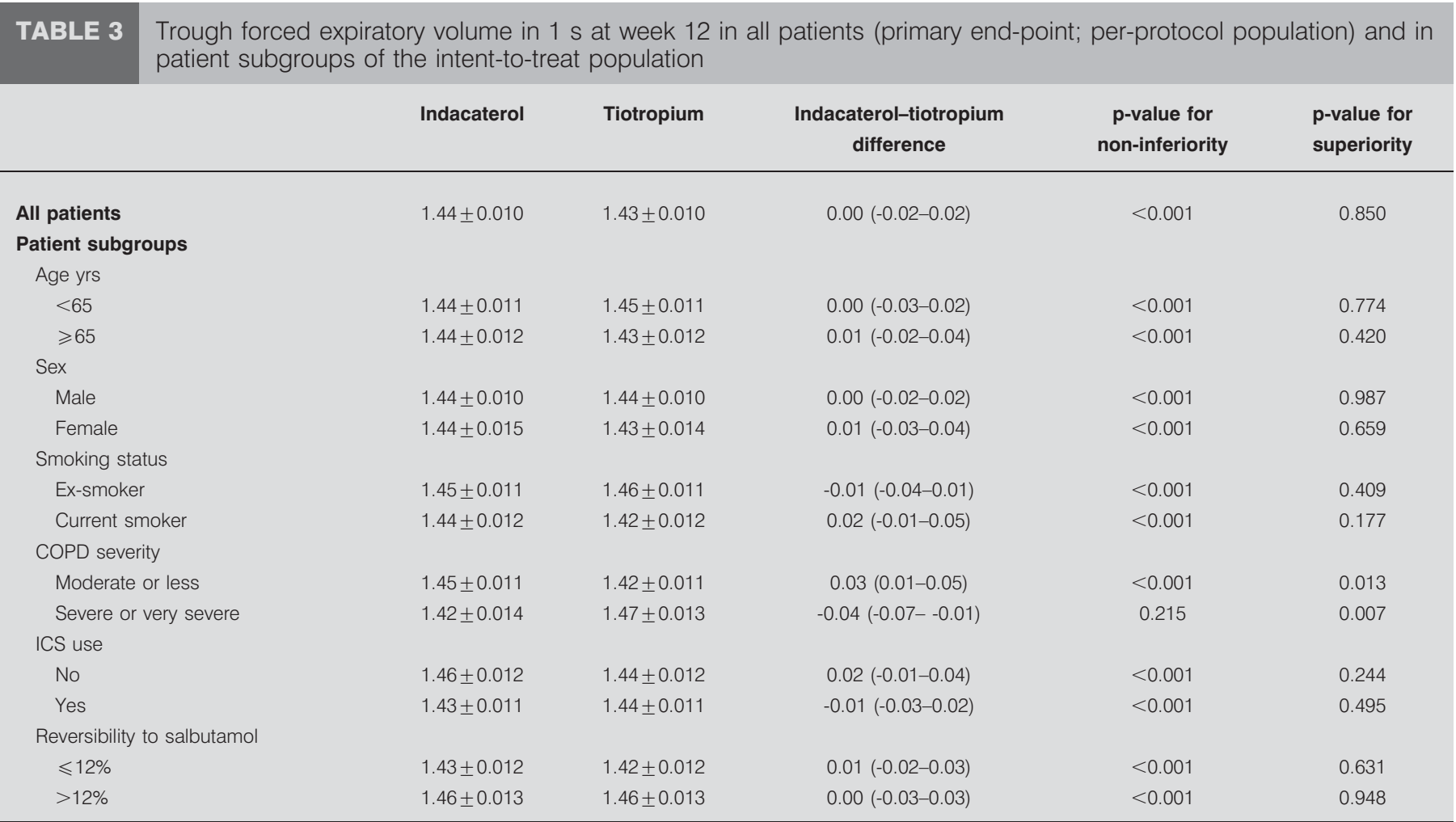

Data are presented as mean \pm SE or mean $(95 \% \mathrm{Cl})$, unless otherwise stated. COPD: chronic obstructive pulmonary disease; ICS: inhaled corticosteroid.

5 min post-dose at week $12(10 \mathrm{~mL}$ in FEV1, $20 \mathrm{~mL}$ in FVC) were not statistically significant, since the immediate bronchodilator effect occurred when the previous doses of treatment were still providing effective bronchodilation, as illustrated by the raw mean change from baseline data in figure 1.

Clinical outcomes: symptoms, health status and diary card data TDI total scores at week 12 showed a greater reduction in dyspnoea with indacaterol than with tiotropium (LSM \pm SE $2.01 \pm 0.178$ and $1.43 \pm 0.178$ points, respectively; $\mathrm{p}<0.001$ for the treatment difference of 0.58 ). Similarly, patients taking indacaterol were significantly more likely (OR 1.49, $\mathrm{p}<0.001)$ to achieve a clinically relevant improvement in dyspnoea (fig. 2).

SGRQ total scores at week 12 demonstrated better health status with indacaterol than with tiotropium (LSM \pm SE $37.1 \pm 0.56$ and $39.2 \pm 0.55$ units, respectively; treatment difference -2.1 , $\mathrm{p}<0.001$ ). Raw mean \pm SD changes (improvements) from baseline were $-5.1 \pm 12.06$ and $-3.0 \pm 11.64$ with indacaterol and tiotropium, respectively. The analysis of the proportion of patients with a clinically relevant improvement in SGRQ total score ( $\geqslant 4$ units) showed that this was statistically significantly more likely to be achieved with indacaterol than with tiotropium (OR 1.43; $\mathrm{p}<0.001$ ) (fig. 2).

Indacaterol-treated patients reduced their daily, daytime and night-time use of rescue salbutamol more than those receiving tiotropium $(p<0.001)$ and had a higher proportion of days without any rescue use $(p=0.004)$ (table 4). Diary data showed that during the 12-week study, the indacaterol- and tiotropium-treated patients had increases from baseline of 2.0 and 1.9, respectively, in the percentage of days with no daytime COPD symptoms, 7.5 and 4.6 in the percentage of nights with no awakenings, and 6.2 and 3.1 in the percentage of days able to undertake usual activities.

\section{Safety}

Adverse events were reported for similar proportions of patients in the two treatment groups (table 5), with the most common events generally reflecting the typical disease characteristics of COPD. The incidence of COPD worsening was 10.7\% with indacaterol and $8.3 \%$ with tiotropium; most cases were mild or moderate in severity with both treatments (92\% (78 out of 85$)$ with indacaterol and $89 \%$ (59 out of 66) with tiotropium).

Serious adverse events occurred in $2.8 \%$ of indacaterol-treated patients and $3.8 \%$ of the tiotropium treatment group. The system organ class most commonly affected was "respiratory, thoracic and mediastinal", reported in 1.0 and $1.3 \%$ of the indacaterol and tiotropium groups, respectively (most of these events were COPD worsening, which includes exacerbations). The only other system organ class with $\geqslant 1.0 \%$ of patients affected in either group was "infections and infestations" (generally respiratory tract infections), reported in $0.9 \%$ and $1.0 \%$ of the indacaterol and tiotropium treatment groups, respectively.

Two patients died during the study, both in the tiotropium treatment group. A 62-yr-old male with a medical history of hypertension died of cardiac arrest due to arrhythmia. The other, a 65-yr-old male, died of septic shock due to bilateral nosocomial pneumonia. Neither death was suspected to be related to the treatment. 

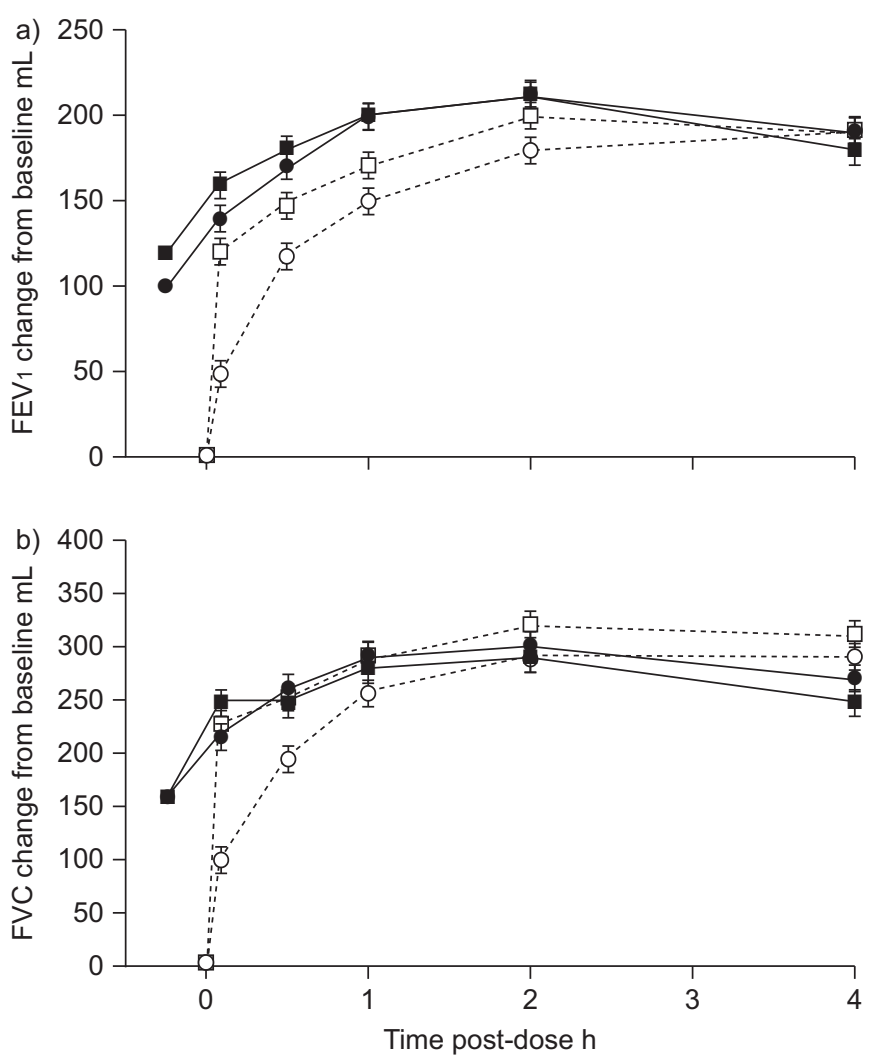

FIGURE 1. Change from baseline in a) forced expiratory volume in $1 \mathrm{~s}$ (FEV 1 ) and b) forced vital capacity (FVC) measured up to $4 \mathrm{~h}$ post-dose on day 1 . $\square$ : indacaterol day 1 ; $\mathbf{\square}$ : indacaterol week 12; 0 : tiotropium day 1 ; $\bullet$ : tiotropium week 12. Data are presented as unadjusted mean \pm SE.

There was little difference between treatment groups for vital signs, plasma potassium and blood glucose, and QTc intervals. Few patients in either group had notable values (table 6).

\section{DISCUSSION}

This study has demonstrated similar efficacy of indacaterol and tiotropium on trough FEV1 after 12 weeks of treatment, with statistical tests establishing non-inferiority of indacaterol compared with tiotropium. Indacaterol had a significantly greater bronchodilator effect than tiotropium during the first hour following dosing. Statistically significantly better results were observed for indacaterol versus tiotropium for the clinical outcomes of dyspnoea, use of as-needed salbutamol and health status.

The magnitude of differences detected as statistically significant varies depending on the size of the study. It is therefore necessary to make a judgement on the clinical relevance of differences between the treatments for the clinical outcomes assessed. This judgement must necessarily be subjective, since reported minimal clinically important differences (MCID) and clinically relevant changes were derived on the basis of differences versus placebo or changes from baseline, not differences between two active treatments $[7,9,10]$.

Dyspnoea and health status are among the most important and robust clinical outcomes in clinical COPD research [14]. Dyspnoea is the most disabling symptom for COPD patients [15]. TDI is a widely used, multidimensional instrument that measures breathlessness related to activities of daily living, and reflects changes in dyspnoea over time and/or in response to treatment [16]. The TDI total scores recorded in this study indicate clinically relevant improvements from baseline in dyspnoea with both treatments, but the additional effect of indacaterol (difference of 0.58 over an established bronchodilator) represents a degree of relief of breathlessness during activities of daily living that may benefit patients with COPD. In addition, patients treated with indacaterol had a $49 \%$ greater likelihood of experiencing a clinically relevant improvement compared with those receiving tiotropium.

The SGRQ assesses changes in health status over time in patients with COPD. The accepted MCID for this measurement is a reduction in total score of 4 units from baseline [10], with a tolerance range of 2.4-5.6 [14]. The mean change for indacaterol exceeded the MCID in this study (-5.1 change from baseline). The result for tiotropium ( -3.0 from baseline) is similar to previous reports over longer periods $(-3.3$ at 6 months [17] and $-2.3--3.3$ units over 4 yrs [18]), although other, smaller studies have reported changes from baseline of 4 units at 6 months [19]. The additional improvement of $>2$ units following treatment with indacaterol seen in this study is close to the tolerance range limit of 2.4 reported by CAzzola et al. [14], and is likely to represent a perceptible difference in patients' health status. Compared with tiotropium, indacaterol-treated patients had a $43 \%$ greater likelihood of achieving a clinically relevant improvement in health status.

Patients with COPD are usually given a short-acting bronchodilator with a fast onset (e.g. salbutamol) to use to relieve acute

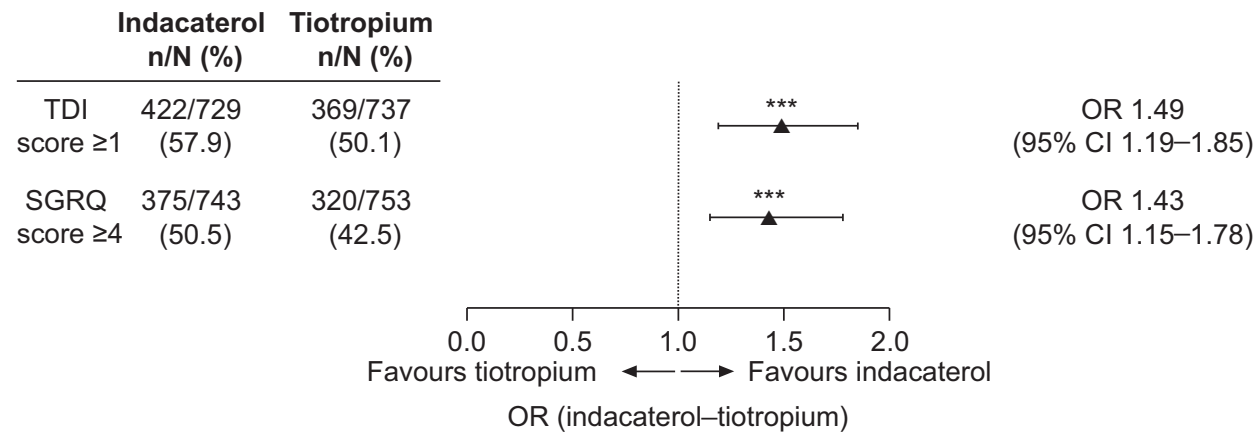

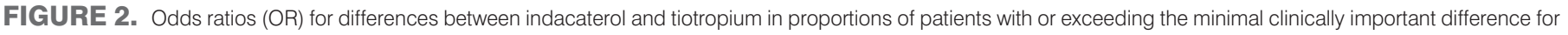
transition dyspnoea index (TDI) total score and St George's Respiratory Questionnaire (SGRQ) score. ***: $\mathrm{p} \leqslant 0.001$ indacaterol versus tiotropium. 
TABLE 4 Use of as-needed salbutamol ("rescue") over 12 weeks

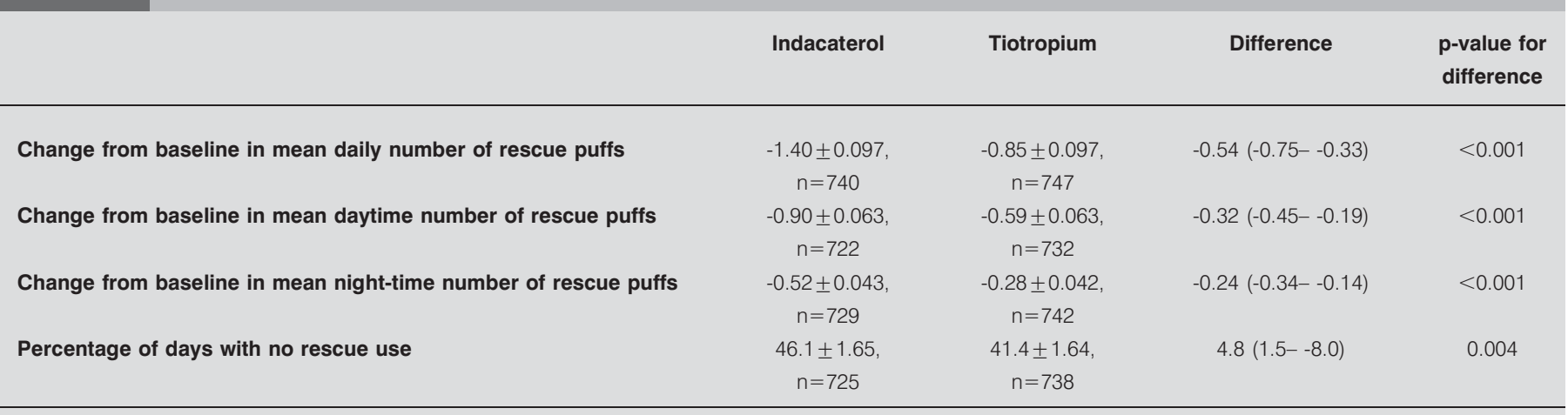

Data are presented as mean \pm SE or mean $(95 \% \mathrm{Cl})$, unless otherwise stated.

symptoms, e.g. exertional dyspnoea. The level of use is reported to correlate with COPD symptoms [20]. Measuring a patient's use of "rescue" medication reflects the effectiveness of maintenance COPD treatment in achieving clinical control. A greater reduction in rescue use with indacaterol over tiotropium was seen (more than half a puff per day), with patients on indacaterol experiencing an additional 5\% of days when no rescue use was needed. This would equate to an additional 17.5 days per year with no requirement for rescue use.

Overall, the effects on clinical outcomes in the present study (indacaterol-tiotropium differences of 0.58 points in TDI total score, -2.1 units in SGRQ total score and -0.54 puffs per day of salbutamol; all $\mathrm{p}<0.001$ ) are of a similar order to those previously reported for tiotropium in comparison with salmeterol (differences at 6 months of 0.78 in TDI total score, -1.6 in SGRQ total score, but no difference in rescue use) [21]. Analogously, indacaterol may represent a further therapeutic advance over tiotropium. The present results also support and expand on those of a previous 6-month study of indacaterol versus tiotropium in which tiotropium was administered unblinded [2].

The closely similar effect of treatments on trough FEV1 indicates that this study is a comparison of equipotent bronchodilator doses and demonstrates true superiority of indacaterol in its effect on clinical outcomes. Indacaterol had

\begin{tabular}{lcc} 
TABLE 5 & $\begin{array}{l}\text { Overall and most commonly occurring adverse } \\
\text { events }^{\#}\end{array}$ & \\
& Indacaterol & Tiotropium \\
\hline Subjects $\mathbf{n}$ & 794 & 799 \\
Any adverse event & $315(39.7)$ & $297(37.2)$ \\
COPD worsening, including exacerbations & $85(10.7)$ & $66(8.3)$ \\
Cough & $37(4.7)$ & $27(3.4)$ \\
Nasopharyngitis & $36(4.5)$ & $37(4.6)$ \\
Headache & $24(3.0)$ & $24(3.0)$ \\
Influenza & $19(2.4)$ & $16(2.0)$ \\
Bronchitis & $16(2.0)$ & $7(0.9)$ \\
\hline
\end{tabular}

Data are presented as $n(\%)$, unless otherwise stated. COPD: chronic obstructive pulmonary disease. ${ }^{\#}: \geqslant 2 \%$ of patients. statistically significantly greater effects on dyspnoea, rescue salbutamol use and health status. However, the mechanistic reasons for these differences are not obvious. There may be differences between indacaterol and tiotropium in their effect on overall lung volume, despite the similarity of FVC results, and it would be interesting to compare effects on inspiratory capacity. The two drugs may have differential bronchodilator effects on small airways, possibly due to regional variation in airway distribution of muscarinic and adrenergic receptors [22], leading to differing non-bronchodilator effects on lung ventilation and pulmonary haemodynamics [23]. A range of non-bronchodilator, generally anti-inflammatory effects has been postulated for long-acting $\beta_{2}$-agonists in COPD [24], although the clinical relevance of these remains to be established. In terms of FEV1, it seems likely that the similar magnitude of response achieved with indacaterol and tiotropium represents the full bronchodilator potential of $\beta_{2}$-agonists and anticholinergics, respectively, and that further improvements will be possible by combining both therapeutic principles, ideally as a fixed combination [25].

\section{TABLE 6 Proportions of patients with notable values for plasma potassium, blood glucose, pulse rate, blood pressure and QTc interval (Fridericia's formula)}

\begin{tabular}{lcc} 
& Indacaterol & Tiotropium \\
\hline & $0 / 778$ & $0 / 776$ \\
Plasma potassium $<\mathbf{3 . 0} \mathbf{~ m m o l} \cdot \mathbf{L}^{-1}$ & $26 / 778(3.3)$ & $20 / 776(2.6)$ \\
Blood glucose $>\mathbf{9 . 9 9} \mathbf{m m o l} \cdot \mathbf{L}^{-1}$ & $2 / 792(0.3)$ & $0 / 799$ \\
High pulse rate & $7 / 792(0.9)$ & $5 / 799(0.6)$ \\
High systolic blood pressure & $6 / 792(0.8)$ & $13 / 799(1.6)$ \\
High diastolic blood pressure & & \\
QTc interval & & \\
Absolute value males/females $>450 / 470 \mathrm{~ms}$ & $23 / 793(2.9)$ & $25 / 799(3.1)$ \\
Absolute value $>500 \mathrm{~ms}$ & $0 / 793$ & $1 / 799(0.1)$ \\
Increase $30-60 \mathrm{~ms}$ & $36 / 788(4.6)$ & $48 / 794(6.0)$ \\
Increase $>60 \mathrm{~ms}$ & $2 / 788(0.3)$ & $0 / 794$ \\
\hline
\end{tabular}

Data are presented as $\mathrm{n} / \mathrm{N}(\%) .{ }^{\#}:>130 \mathrm{bpm}$, or $\geqslant 120 \mathrm{bpm}$ and $\geqslant 15 \mathrm{bpm}$ increase from baseline; $?>200 \mathrm{mmHg}$, or $\geqslant 180 \mathrm{mmHg}$ and $\geqslant 20 \mathrm{mmHg}$ increase from baseline; ${ }^{+}:>115 \mathrm{mmHg}$, or $\geqslant 105 \mathrm{mmHg}$ and $\geqslant 15 \mathrm{mmHg}$ increase from baseline 
The small differential in bronchodilator effect in patient subgroups of differing disease severities may also reflect mechanistic differences between the two bronchodilators, or may reflect shortcomings in FEV1 as a single measure of treatment effect in a heterogeneous condition such as COPD [15]. While potentially interesting, the actual differences of 30$40 \mathrm{~mL}$ are very small and well below the 100-140 mL level of clinical relevance [14]. Previously, indacaterol has been reported to have a similar effect in patients with differing COPD severities [12].

The present study has advantages over previous reported comparisons of indacaterol and tiotropium, being larger and more robustly blinded than the previous 6-month study with unblinded tiotropium [2], and larger and longer than the previous 2-week double-blind study [3]. In terms of blinding of the present study, study drugs were dispensed by a third party who was not otherwise involved in the study and, because this was a parallel-group study, no patient received both active and placebo tiotropium.

The improvements in clinical outcomes observed during treatment with indacaterol were obtained without any increase in side-effects or other safety signals compared with tiotropium. Comparable safety of these two treatments was also observed in the previous 6-month open-label comparison [2], and indacaterol has previously demonstrated an acceptable tolerability and safety profile with the $300 \mu \mathrm{g}$ and the abovetherapeutic $600 \mu \mathrm{g}$ doses given for up to $1 \mathrm{yr}$ compared with placebo [12]. Overall, these results present a reassuring safety profile for this new bronchodilator.

In conclusion, we believe that indacaterol may provide more symptomatic benefit than tiotropium and, as such, extends the therapeutic options for patients with COPD.

\section{SUPPORT STATEMENT}

This study was sponsored by Novartis.

\section{CLINICAL TRIAL}

This study is registered at www.ClinicalTrials.gov (identifier: NCT00900731).

\section{STATEMENT OF INTEREST}

Statements of interest for all authors and the study itself can be found at www.erj.ersjournals.com/site/misc/statements.xhtml

\section{ACKNOWLEDGEMENTS}

We thank the patients and staff at the participating centres in the study. We also thank S. Filcek (professional medical writer funded by Novartis; Acumed, Tytherington, UK,) and D. Young (Novartis, Horsham Research Centre, West Sussex, UK) who assisted in the preparation of the manuscript.

\section{REFERENCES}

1 Global Initiative for Chronic Obstructive Lung Disease. Global Strategy for the Diagnosis, Management and Prevention of COPD. 2010. www.goldcopd.org

2 Donohue JF, Fogarty C, Lötvall J, et al. Once-daily bronchodilators for chronic obstructive pulmonary disease: indacaterol versus tiotropium. Am J Respir Crit Care Med 2010; 182: 155-162.
3 Vogelmeier C, Ramos-Barbon D, Jack D, et al. Indacaterol provides 24-hour bronchodilation in COPD: a placebo-controlled blinded comparison with tiotropium. Respir Res 2010; 11: 135.

4 Global Initiative for Chronic Obstructive Lung Disease. Global Strategy for the Diagnosis, Management and Prevention of COPD. 2007. Available from: www.goldcopd.org/Guidelines/guidelinesglobal-strategy-for-diagnosis-management-2007-3.html

5 Miller MR, Hankinson J, Brusasco V, et al. Standardisation of spirometry. Eur Respir J 2005; 26: 319-338.

6 Mahler DA, Weinberg DH, Wells CK, et al. The measurement of dyspnoea. Contents, interobserver agreement, and physiologic correlates of two new clinical indexes. Chest 1984; 85: 751-758.

7 Witek TJ Jr, Mahler DA. Minimal important difference of the transition dyspnoea index in a multinational clinical trial. Eur Respir J 2003; 21: 267-272.

8 Jones PW, Quirk FH, Baveystock CM, et al. A self-complete measure of health status for chronic airflow limitation. The St. George's Respiratory Questionnaire. Am Rev Respir Dis 1992; 145: 1321-1327.

9 Jones PW. Interpreting thresholds for a clinically significant change in health status in asthma and COPD. Eur Respir J 2002; 19: 398-404.

10 Jones PW. St. George's Respiratory Questionnaire: MCID. COPD 2005; 2: 75-79.

11 Feldman G, Siler T, Prasad N, et al. Efficacy and safety of indacaterol $150 \mu \mathrm{g}$ once-daily in COPD: a double-blind, randomised, 12-week study. BMC Pulm Med 2010; 10: 11.

12 Dahl R, Chung KF, Buhl R, et al. Efficacy of a new once-daily longacting inhaled $\beta_{2}$-agonist indacaterol versus twice-daily formoterol in COPD. Thorax 2010; 65: 473-479.

13 Barr RG, Bourbeau J, Camargo CA, et al. Tiotropium for stable chronic obstructive pulmonary disease: a meta-analysis. Thorax 2006; 61: 854-862.

14 Cazzola M, MacNee W, Martinez FJ, et al. Outcomes for COPD pharmacological trials: from lung function to biomarkers. Eur Respir J 2008; 31: 416-469.

15 Celli BR, Cote CG, Marin JM, et al. The body-mass index, airflow obstruction, dyspnoea, and exercise capacity index in chronic obstructive pulmonary disease. N Engl J Med 2004; 350: 1005-1012.

16 Mahler DA. Mechanisms and measurement of dyspnoea in chronic obstructive pulmonary disease. Proc Am Thorac Soc 2006; 3: 234-238.

17 Casaburi R, Mahler DA, Jones PW, et al. A long-term evaluation of once-daily inhaled tiotropium in chronic obstructive pulmonary disease. Eur Respir J 2002; 19: 217-224.

18 Tashkin DP, Celli B, Senn S, et al. A 4-year trial of tiotropium in chronic obstructive pulmonary disease. N Engl J Med 2008; 359: 1543-1554.

19 Brusasco V, Hodder R, Miravitlles M, et al. Health outcomes following treatment for six months with once daily tiotropium compared with twice daily salmeterol in patients with COPD. Thorax 2003; 58: 399-404.

20 Partridge MR, Miravitlles M, Ståhl E, et al. Development and validation of the Capacity of Daily Living during the Morning questionnaire and the Global Chest Symptoms Questionnaire in COPD. Eur Respir J 2010; 36: 96-104.

21 Donohue JF, van Noord JA, Bateman ED, et al. A 6-month, placebo-controlled study comparing lung function and health status changes in COPD patients treated with tiotropium or salmeterol. Chest 2002; 122: 47-55.

22 Barnes PJ. Distribution of receptor targets in the lung. Proc Am Thorac Soc 2004; 1: 345-351.

23 Ashutosh K, Dev G, Steele D. Nonbronchodilator effects of pirbuterol and ipratropium in chronic obstructive pulmonary disease. Chest 1995; 107: 173-178.

24 Johnson M, Rennard S. Alternative mechanisms for long-acting $\beta_{2}-$ adrenergic agonists in COPD. Chest 2001; 120: 258-270.

25 Van Noord JA, Buhl R, LaForce C, et al. QVA149 demonstrates superior bronchodilation compared with indacaterol or placebo in patients with chronic obstructive pulmonary disease. Thorax 2010; 65: 1086-1091. 